BULGARIAN ACADEMY OF SCIENCES

CYBERNETICS AND INFORMATION TECHNOLOGIES • Volume 15, No 4

Sofia $\bullet 2015$

Print ISSN: 1311-9702; Online ISSN: 1314-4081

DOI: $10.1515 /$ cait-2015-0061

\title{
Modeling and Topological Properties of a V2I Sub Network in VANET Based on a Complex Network
}

\author{
Hong Zhang ${ }^{1}$, Li He ${ }^{2}$ \\ ${ }^{1}$ Transportation Institute - Inner Mongolia University, Hohhot, Inner Mongolia 010021, China \\ ${ }^{2}$ Department of Road and Bridge Engineering - Guizhou Polytechnic College of Communications, \\ Guiyang, Guizhou 550008, China \\ Emails: zhang2001hong@126.com hust_heli@126.com
}

Abstract: Vehicles to Infrastructure (V2I) communicate with each other in a Vehicular Ad hoc NETwork (VANET) that can be represented as a complex network. In them much interest has been attracted towards the topological properties and structure recently, and many studies focus the attention on it, in particular V2I sub network. V2I is an important basic part of the future intelligent transportation, which transfers information through a wireless communication network. Analyzing the topological properties would help understand the VANET system structure and reveal the essence of the network. In this paper we propose a V2I model in VANET based on the complex network theory, analyzing the degree distribution. VANET topology characteristics are designed and discussed. The simulation results further illustrate the efficiency and applicability of the proposed approach.

Keywords: Intelligent transportation, topological characteristics, complex network, VANET, Vehicle to Infrastructure.

\section{Introduction}

The networks are generally complex, and the complexity of the networks would be viewed from different perspectives. Complex networks have been studied extensively in the last decade, in particular, social networks, transportation networks, WWW, co-authorship networks and so on [1]. Most classes of networks exhibit topological characteristics in common, such as the small world feature or scale free degree distribution, which is revealed by a dynamical mechanism known 
as rewire attachment and preferential attachment. Many complex systems have both dynamical aspects. In these properties; the main focus is on the macroscopic models or the local topological aspects, e.g., the degree, clustering coefficient, shortest path length and those distributions. In the present Vehicular Ad hoc NETwork (VANET) model [2], the nodes denote vehicles which have the ability to deliver packets, and the edges denote vehicles wireless linkage. The Connected Vehicles are focused on localized Vehicle-to-Vehicle (V2V), Vehicle-to-Infrastructure (V2I) and Vehicleto-Device (V2D) systems to support safety, mobility and environmental applications using vehicle Dedicated Short Range Communications (DSRC) and/or Wireless Access for Vehicular Environments (WAVE). Examples of crash scenarios and V2I application are shown in Fig. 1. As the communication link between the vehicles and the roadside infrastructure might exist for only a short amount of time, the IEEE $802.11 \mathrm{p}$ amendment defines a way to exchange data through this link without the need to establish a basic service set, and thus, without the need to wait for the association and authentication procedures to complete before exchanging data. For this purpose, the vehicles may start sending and receiving data frames as soon as they arrive on the communication channel.

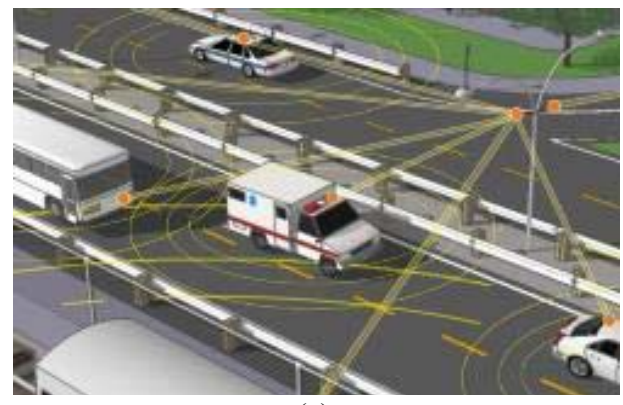

(a)

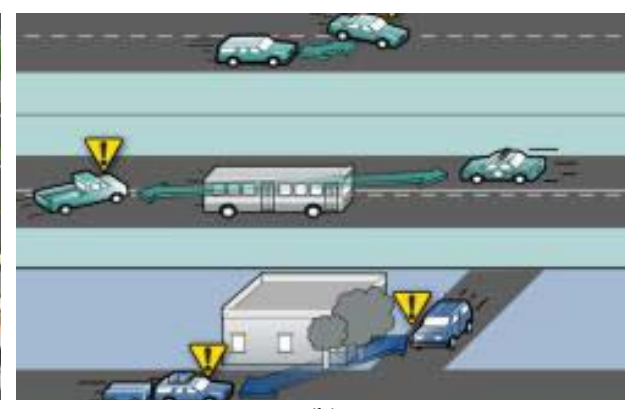

(b)

Fig. 1. Visual representation of V2V communication (a), examples of crash scenarios (b)

VANET has the following five characteristics:

(i) Mobility. VANET is composed of mobile nodes and fixed nodes. The mobile node's geographical location may change at any time based on the variable speed movement. So the topology of the network changes, but does not affect the overall operation of the network. In fact, it is the biggest difference between VANET and a fixed structure network.

(ii) Wireless. The wireless channel is not stable, vulnerable to interference and monitoring, security has become an important research topic.

(iii) Multi hop [3]. Since the wireless transmit power and signal coverage is limited, while it needs to communicate with other nodes outside the coverage, it must be multi hop to complete with an intermediate point.

(iv) Self-organization. Each node moves quickly, independent of the composition of the network. All nodes are coordinated according to the distributed algorithm. This is the important reason VANET has good prospects for application.

(v) High dynamic [4]. The mobile nodes can open and close the network according to their own needs when the network is affected by antenna coverage. VANET topology also changes quickly. 
VANET, combined with Intelligent Transportation Systems (ITS), Global Positioning System (GPS) technologies to identify the vehicle location, which can guarantee traffic safety [5], prevent traffic congestion and guide the vehicles to achieve an autopilot [6].

\section{Assumption and model}

The recent study of the complex networks has directed most interests to the modelling and understanding of various complex networks, especially the relations between the complexities of the network topology and the behaviours of the network dynamics. To describe the common properties and characteristic features of VANET, graphs in mathematics are introduced in the paper. VANET can be viewed as a graph consisting of nodes connected by edges in which the vehicles denote nodes. When the vehicles communicate with other vehicles, they first select for each other the nearest vehicles to connect, similar to a Scale Free network model. By its very nature, VANET is a wireless network, or more precisely a network of heterogeneous vehicles, sub networks, such as V2I, V2V and V2D, which are mutually interconnected in many different ways. V2I are used to connect each other within a relatively small local area. VANET is a typical complex network with large numbers of nodes and edges, it processes huge amounts of information data growing rapidly and continuously [7]. To model this extremely huge and complex network, or a part of it, topology generators are usually used.

For simulation, we generated a network by the following Algorithm:

Step 1. Start from a fully-shaped network with small size $m_{0} \geq 1$, in which each node is connected to its neighbours, where there are $m_{0}\left(m_{0}-1\right) / 2$ edges (see Fig. 1) Introduce one new node to the existing network each time, and the new node is connected to $m\left(m_{0} \geq m \geq 1\right)$ existing nodes in the network simultaneously [8].

Step 2. The above incoming new node is connected to each of the $m$ existing nodes, the node $i$ of degree $k$ according to the preferential attachment is given as the probability

$$
\prod_{i} k=\frac{k_{i}}{\sum_{j} k_{j}} .
$$

At the $t$-th step of the node adding process, the total number of the existing nodes is given, where $N=m_{0}+t, t=0,1,2, \ldots, n$.

Here, at Step 2, the preferential attachment probability is proportional to $k_{i}+1$, so that the isolated node with $k_{i}=0$ has a non-zero probability to acquire new edges; otherwise it will be an isolates node. If the initial network is connected, then +1 in (1) is not needed [9]; otherwise, comparing to a large number of $N$, this +1 does not change much in the formula anyway. Clearly, after $t$ steps, the total possible number of nodes and edges has been determined beforehand which may be equal to $N=t+m_{0}$ nodes and $m t+m_{0}\left(m_{0}-1\right) / 2$ edges.

The node degree $k$ is the number of nodes it is directly linked to, and its strength can be viewed as an indication of its centrality. The average $k$ is expressed 
as $\langle k\rangle$, and $p(k)$ describes its degree distribution where a randomly selected node degree is exactly the probability of $k$, also equal to the degree $k$ of number of nodes in the total number of network nodes [10]. Traditionally, for a network $G$, the degree distribution could be denoted as $p(k)=N_{k} / N$.

\section{Theoretical considerations}

Start with a network of $n_{0}$ nodes. At each step, one new node is being added along with $m_{1}$ new edges connecting to $m$ existing nodes, where the probability $q$ of an existing node $i$, being chosen by the new for connection is [11]

$$
\left(\frac{\partial k_{i}}{\partial t}\right)_{i i}=\frac{m_{1} q}{m+t} \frac{k_{i}+\alpha}{\sum_{j \in \Re} k_{j}+\alpha},
$$

where $\alpha$ is an adjustable parameter. Next adding $m_{2}$ new edges connecting to $m_{1}$ nodes with probability of $r$, there is obtained:

$$
\begin{gathered}
\left(\frac{\partial k_{i}}{\partial t}\right)_{i i i}=\frac{m_{2} r}{m+t p}\left[\frac{1}{N_{\Re}(t)}+\left(1-\frac{1}{N_{\Re}(t)}\right) \frac{k_{i}+\alpha}{\sum_{j \in \Re} k_{j}+\alpha}\right], \\
\frac{\partial k_{i}}{\partial t}=\left[\frac{q m_{1}}{c}+\frac{r m_{2}\left(q+m_{0} p-p\right)}{\left(q+m_{0} p\right) c}+\frac{s m_{3} p}{\left(q+m_{0} p\right) c}+\frac{2 u m_{4}}{c}\right] \frac{k_{i}}{t}+ \\
+\left[\frac{q m_{1} \alpha}{c}+\frac{r m_{2}}{q+m_{0} p}+\frac{r m_{2}\left(q+m_{0} p-p\right)}{\left(q+m_{0} p\right) c}+\frac{s m_{3} p \alpha}{\left(q+m_{0} p\right) c}+\frac{2 s m_{3}}{q+m_{0} p}+\frac{2 u m_{4} \alpha}{c}\right] \frac{1}{t}, \\
a=\frac{q m_{1}}{c}+\frac{r m_{2}\left(q+m_{0} p-p\right)}{\left(q+m_{0} p\right) c}+\frac{s m_{3} p}{\left(q+m_{0} p\right) c}+\frac{2 u m_{4}}{c}, \\
b=\frac{q m_{1} \alpha}{c}+\frac{r m_{2}}{q+m_{0} p}+\frac{r m_{2}\left(q+m_{0} p-p\right)}{\left(q+m_{0} p\right) c}+\frac{s m_{3} p \alpha}{\left(q+m_{0} p\right) c}-\frac{2 s m_{3}}{q+m_{0} p}+\frac{2 u m_{4} \alpha}{c} \text { so, } \\
\frac{\partial k_{i}}{\partial t}=\frac{\left(a k_{i}+b\right)}{t} .
\end{gathered}
$$

The initial conditions are:

$$
a \neq 0, k_{i}\left(t_{i}\right)=m_{1}, k_{i}(t)=-\frac{a}{b}+\left(m_{1}+\frac{b}{a}\right)\left(\frac{t}{t_{i}}\right)^{a} .
$$

The robability density function of $t_{i}$ is $P_{i}\left(t_{i}\right)=\frac{1}{3 m+t(1+2 p)}$ and so

$$
P\left(k_{i}(t)<k\right)=P\left[t_{i}>\left(\frac{m_{1}+b / a}{k+b / a}\right)^{1 / a} t\right]=1-\frac{1}{[3 m+t(1+2 p)]}\left(\frac{m_{1}+b / a}{k+b / a}\right)^{1 / a} t .
$$

Assume that $P(k)=\frac{\partial\left\{P\left[k_{i}(t)<k\right]\right\}}{\partial k}$, we get 


$$
P(k)=\frac{1}{a[3 m+t(1+2 p)]}\left(m_{1}+\frac{b}{a}\right)^{1 / a}\left(k+\frac{b}{a}\right)^{-\gamma},
$$

where $\gamma=1+1 / a$.

Theorem 1. The node degree distribution of the Scale Free network model is given by [3]

$$
P(k)=\frac{\partial P\left(k_{i}(t)<k\right)}{\partial k}=2 \frac{m^{2} t}{t+m_{0}} k^{-\gamma} \approx 2 m^{2} k^{-\gamma} .
$$

This is the power law, in the form of $k^{-\gamma}$, with $2<\gamma<3$.

According to the previous research results $\gamma=2 \sim 3$ [12], Equation (4) needs to satisfy the following conditions:

$$
\left\{\begin{array}{c}
\left(m_{1}+b / a\right)>0 \\
a<1
\end{array}\right.
$$

VANET consists of a large number of interconnected V2I. Each V2I may be considered as a Stub domain or a Transit domain. A Transit domain can be a MAN or a WAN. Typically, a Stub domain consists of V2I networks or some other interconnected LAN, depending on the respective domain of some parts of the domain, to carry out information processing and communications [13]. The structure of VANET at V2I level is illustrated by Fig. 2. When a new Autonomous System is being added into the network, it creates a certain number of edges connecting the existing nodes. On the other hand, there may also be interconnections between the already existing nodes, for example, due to the consideration of having redundancy for having a fault tolerance or for avoiding a possible traffic congestion. The structure of V2I at Autonomous Systems level is shown in Fig. 2.

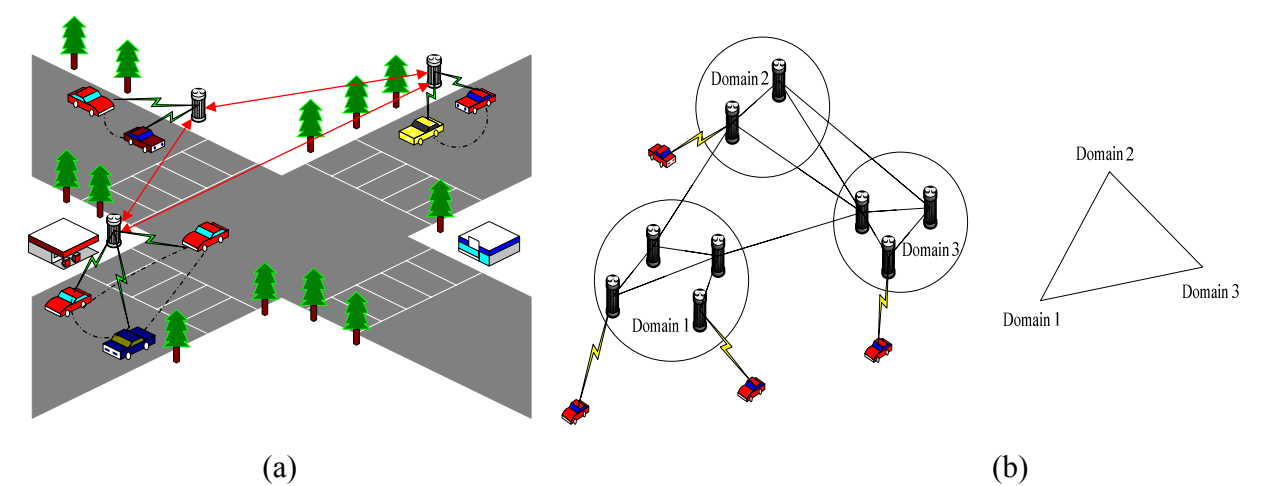

Fig. 2. Structure of V2I at Autonomous Systems level (a); VANET topology level (b)

To present and model V2I network, a nature way is to put the framework into a complex setting, in which a node represents an individual and the edge represents the interaction between the two of them as shown in Fig. 3, which has $N=245$. 


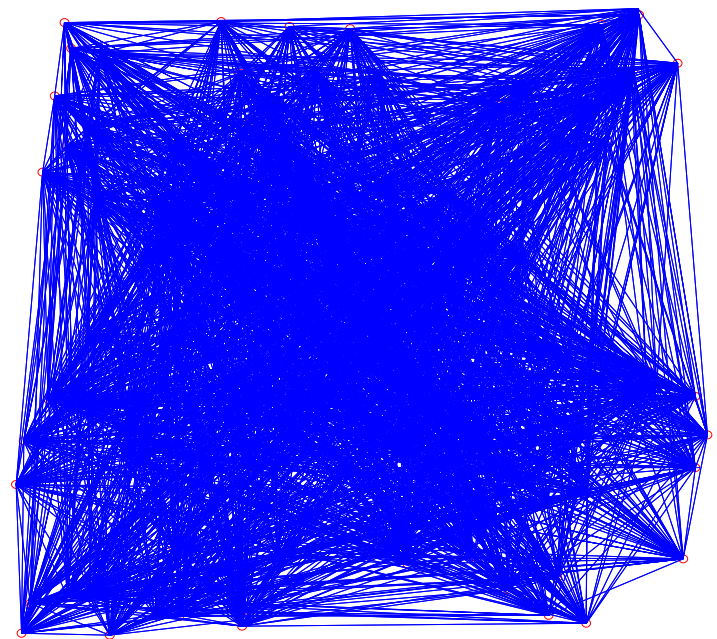

Fig. 3. V2I network topology

\section{Example and simulation analysis}

This paper takes the taxi network in Wuhan, Hubei province as a research object. The number of nodes of V2V network in Guanggu intersection change with the a time step $t$. In order to obtain a better performance, we should make some assumption to the practical network in Wuhan: V2V sub network contains two basic elements, i.e. vehicles and wireless connection, which makes the abstraction of the network to be an undirected network; the vehicles are regarded as a mobile node, which connect with one node at this moment and may disconnect at the next moment.

Based on the data of $\mathrm{V} 2 \mathrm{~V}$ network and the assumptions above given, 245 nodes are jointed experimentally. Each vehicle, using a mobile phone as a mobile terminal is connected to each other.

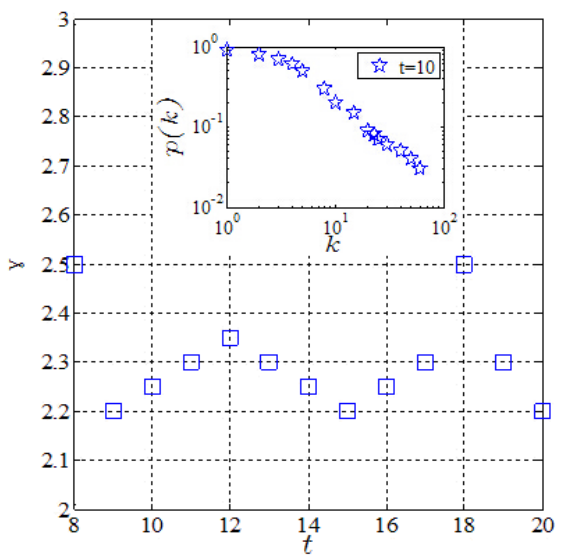

Fig. 4. The relationship of $\gamma$ and $t$ in a V2I network 
V2I sub networks may have a Scale Free characteristic like a random graph, i.e. the degree distribution follows a power law distribution. For the interval 8:0020:00 time, the simulations also show that the time scale for VANET to reach consensus is as shown in Fig. 4, where the exponent has a maximum size at 8:00 and 18:00, and maybe this period of time is the peak morning and evening commute.

In VANET, a few nodes have a large number of edges, called hubs, and they tend to connect with each other, leading to a structure called a rich club [14].

The rich club phenomenon in Autonomous Systems of size $N$ can be described by the connectivity index $\phi(r / N)$ of its first $r$ biggest nodes defined by the ratio of the number $M$ of their existing edged ones versus the number $r(r-1) / 2$ of all possible edged among them:

$$
\phi(r / N)=\frac{2 M}{r(r-1)} .
$$

If $\phi(r / N)=1$, then the first $r$ biggest nodes compose a fully connected sub network, as shown in Fig. 5.

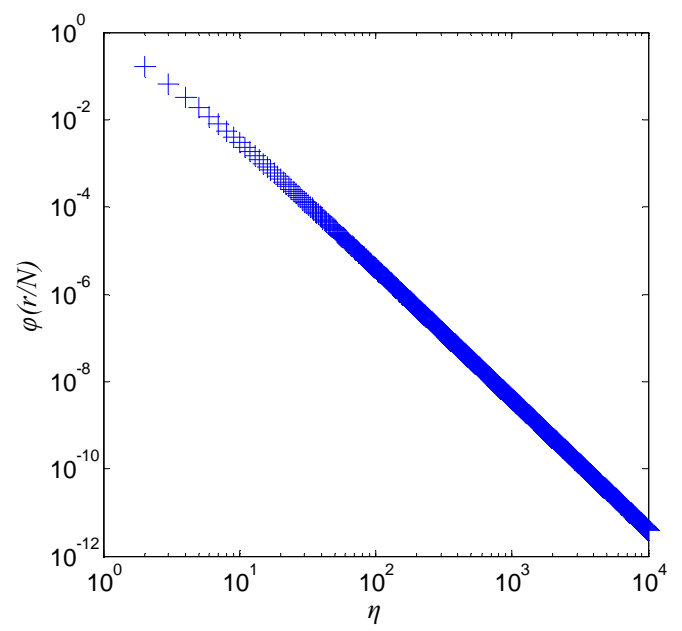

Fig. 5. Rich club phenomenon of V2I network at the Autonomous System level

Reportedly, some real data have verified that the connectivity index $\phi(r / N)$ follows a power law form; $\phi(r / N) \sim r^{-\eta}$ with $\eta=1.1 \pm 0.2$ for the Autonomous System levels and $\eta=1.8 \pm 0.2$ for the router levels of V2I sub network.

As just mentioned, in V2I sub network hubs are well interconnected. Because the hubs are big nodes of higher degrees, it is quite phenomenal that most neighbours are of a hub typically small degrees. The phenomenon can be described by the so-called assortativity coefficient.

The assortativity coefficient of a network is defined by [15] 


$$
r=\frac{M^{-1} \sum_{i} j_{i} k_{i}-\left[M^{-1} \sum_{i} \frac{1}{2}\left(j_{i}+k_{i}\right)\right]^{2}}{M^{-1} \sum_{i} \frac{1}{2}\left(j_{i}^{2}+k_{i}^{2}\right)-\left[M^{-1} \sum_{i} \frac{1}{2}\left(j_{i}+k_{i}\right)\right]^{2}},
$$

where $k_{i}$ and $j_{i}$ are the degrees of the end nodes of edges $i$, and $M$ is the total number of edges in the V2I sub network. If $r>0$ then the network is assortative; if $r<0$, then it is disassortative.

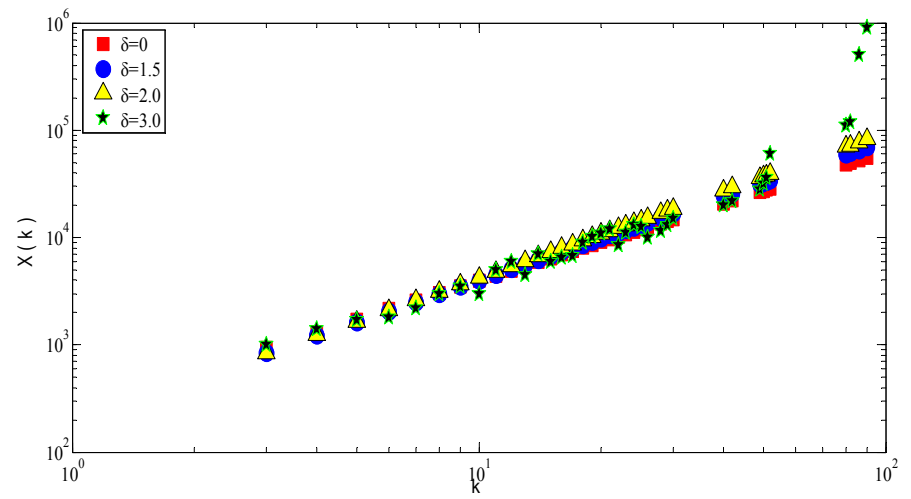

Fig. 6. The log-log relationship of $k$ and $X(k)$

Based on careful analysis of the network, similar to $p(k) \sim k^{-\gamma}$, the information flow $X(k)$ and $k$ has a power law distribution $X(k) \sim k^{\delta}$. Fig. 6 plots the resulting curves calculated based on the real taxi network data on May 26, 2014. It shows that all these parameters are changing rather rapidly although the nodes' degree grows very slowly (for example, the information flow of nodes increases from 1000 on $k=2$ to 90000 on $k=94$ when $\delta=1.5$ ). As $\delta$ is $0,1.5,2.0$ respectively, the power law characteristic is almost constant, while at $\delta=3.0$ it deviates from power law when $k>85$.

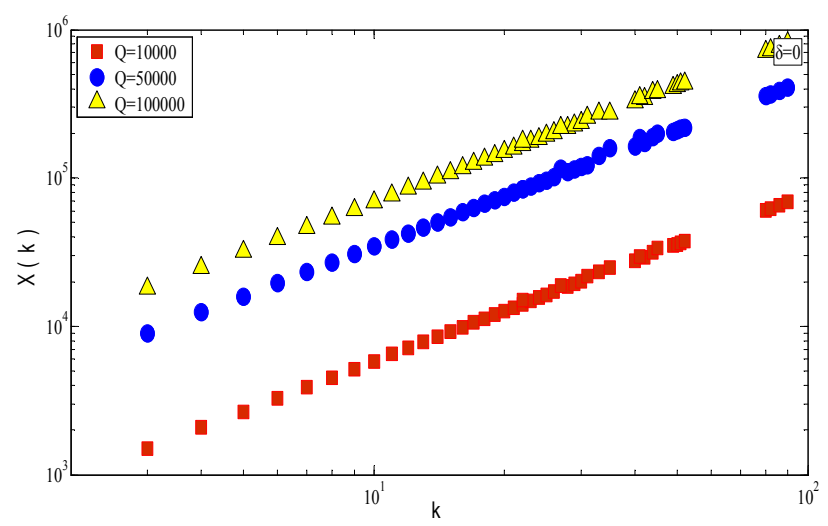

Fig. 7. The log-log relationship of $k$ and $X(k)$ ( $Q$ is variable) 
We assume that $\delta=0, Q$ denotes the total information flow between the nodes. Fig. 7 shows the power law is regular between $k$ and $X(k)$.

As seen above, there are some fundamental characteristics that are never changed, or basically do not change, during the evolution of V2I sub network, such as the power law distribution of the nodes degrees.

\section{Dynamical behaviors of the topological characteristics}

Recently, the topological characteristics have been extensively used to evaluate and differentiate models. In most of the cases, however, such comparisons are based only on one snapshot data of the network. As an open network, V2I sub network continuously evolves with new nodes and new links joining the network while some existing nodes and links disappear from the network from time to time. As a result, the topological characteristics of V2I sub network also evolve with time. A recent work [16] suggested that the dynamical behaviors of the topological characteristics can be useful when comparing and evaluating V2I models.

Fig. 8 shows the number nodes as a function of time on October 10, 2014. From the figure we can see that the number of nodes in the V2I sub network increases almost linearly, albeit with some fluctuations.

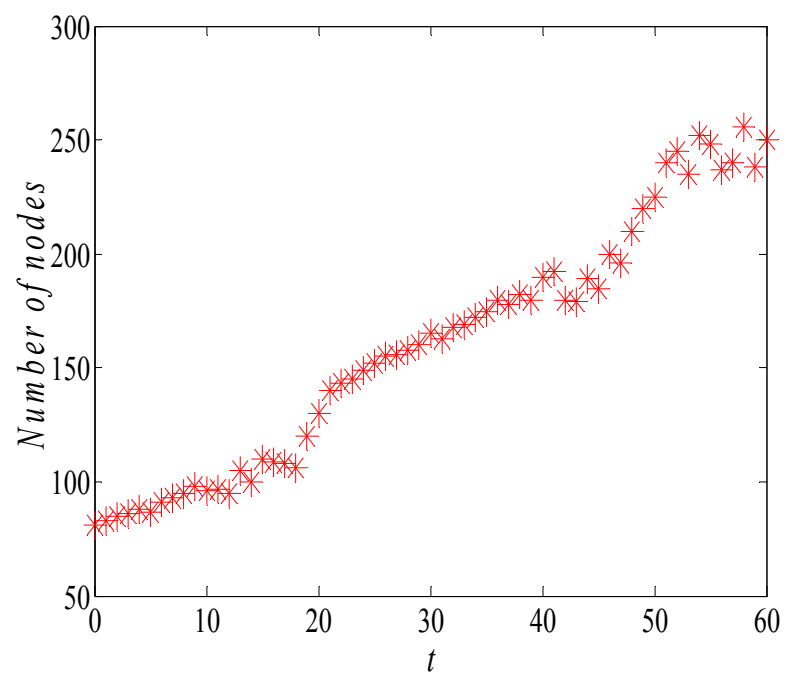

Fig. 8. Number of nodes

If the dynamical behaviors of the model capture the V2I sub network well, the task of modeling VAENT is still quite far from being completed, greatly challenging future investigations.

Fig. 9 shows the average distance for the nodes in a V2I sub network. We can see that the value of the average distance is remarkably small, showing a typical small world connected, rather different from the previous observation. 


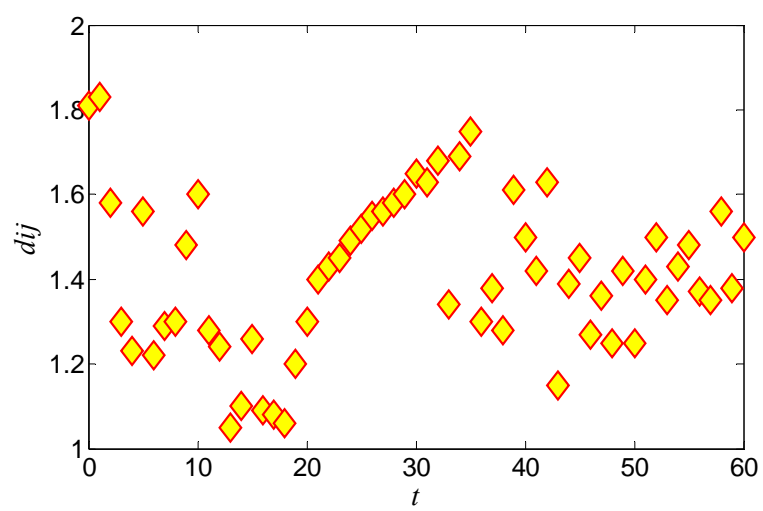

Fig. 9. Average distance for nodes

Definition 1. The information centrality of a graph $G$ of size $N$ is defined to be the mean information flow rate over the network:

$$
E(G)=\frac{1}{N(N-1)} \sum_{i \neq j \in G} \frac{1}{d_{i j}}=\frac{1}{N(N-1)} \sum_{i \neq j \in G} \varepsilon_{i j},
$$

where the network efficiency $\varepsilon_{i j}$ of the channel information transmission [17] between node $i$ and $j$ is defined to be inversely proportional to the shortest distance $d_{i j}$. If there are no edges in the graph, then $d_{i j}=\infty$, so $\varepsilon_{i j}=0$.

The concept of information centrality is very useful in, for example, finding the structure of a network.

For example, one model uses an undirected weighted network $G$ to model a communication or a transportation system, in which the weight on the edge between node $i$ and $j$ is denoted by $e_{i j}\left(e_{i j} \in[0,1]\right)$. The bigger the $e_{i j}$ is, the more efficient the data traffic is. Thus, the $N \times N$ coupling matrix $\left\{e_{i j}\right\}$ describes the efficiency of communications among the nodes. Initially, set all $e_{i j}=1$. Let $L_{i}(t)$ be the loading of node $i$ at time $t$, representing the number of efficiency optimal paths that pass through node $i$. Here, for all paths that pass through the edge between the node pair $(i, j)$, the efficiency optimal path is the one that maximizes $e^{*}=\sum\left(1 / e_{k}\right)^{-1}$, and it is denoted by $e_{\max }^{*}$. The capacity of the node $i$ is defined to be $C_{i}=\alpha \times L_{i}(0)$, where the constant $\alpha \geq 1$ is a tolerance parameter.

When the model is applied to an ER network and a BA network of the same size with $N=1000$ and $M=5000$, comparison is given in Fig. 10, in which both networks have their threshold values $\alpha_{C}$ : where $\alpha<\alpha_{C}$, the network collapses, with $E(G)<0.28$. But the ER network is much smaller than that of the BA network, showing that ER networks are more robust [18] than the BA networks in resisting cascading failures. Besides, for both ER and BA networks, the node removal scheme based on loadings is easier to create cascading failures [19] than the random removal scheme. 


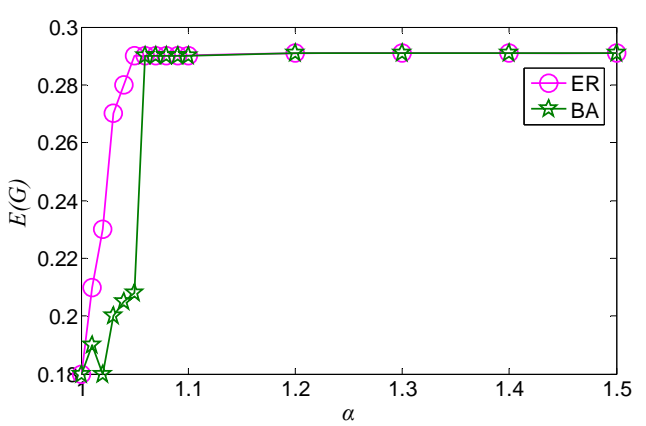

(a)

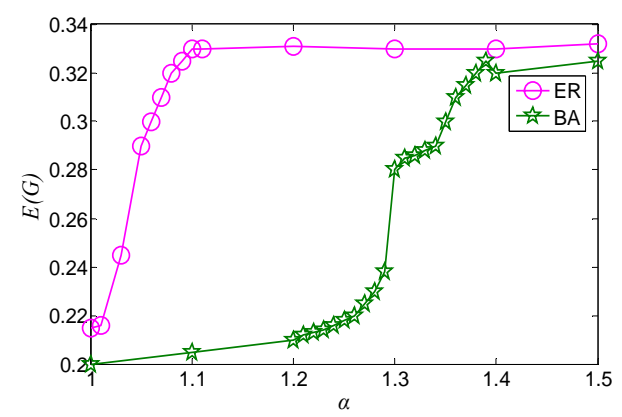

(b)

Fig. 10. Average efficiency $E(G)$

Moreover, as $\alpha$ decreases, this decrease of $E(G)$ becomes more severe; on the contrary, a random failed node removal does not have prominent effects. It is clear that as the tolerance value $\alpha$ increases, the steady-state of $E(G)$ approaches the initial values, meaning that if the tolerance is relaxed, then loading will become less serious, consistent with the common intuition.

\section{Conclusion}

We have introduced complex network theory models that are suitable for characteristics of V2I sub networks, in which the nodes denoted the vehicles in VANET and the edges denoted the wireless communication between vehicles. According to the assumptions of the network models, we calculated the relation parameters, such as degree distribution, rich club phenomenon and information flow distribution that indicates the power law properties in V2I network.

In the dynamical behaviors of the topological characteristics, we found that the number of nodes in V2I sub network increases almost linearly, albeit with some fluctuations, and the average distance of nodes is small which shows some small world network characteristics. Compared with ER network and BA network, we found that ER networks are more robust than the BA networks in resisting cascading failures. Besides, for both ER and BA networks, the node removal scheme based on loadings is easier when creating cascading failures than the random removal scheme.

Acknowledgment: The research is sponsored by the Scientific Research Fund of Hubei Provincial Education Department (B2015372). We am grateful to the anonymous reviewers for their insightful comments and recommendations.

\section{References}

1. W a t t s, D. J. The "New" Science of Networks. - Annual Review of Sociology, Vol. 30, 2004, pp. 243-270.

2. S h e n, M., P. P e n g, P. Z h a o. A Suitable for the Research of VANET Networking Method of Urban Transportation. - Microelectronics \& Computer, Vol. 31, September 2014, No 9 , pp. 105-108. 
3. Shahidi, R., M. H. Ahmed. Probability Distribution of End-to-End Delay in a Highway VANET. - IEEE Commun. Lett., Vol. 18, March 2014, No 3, pp. 443-446.

4. Urquiza, L., C. Tripp, I. Martin, M. A guil ar. Propagation and Packet Error Models in VANET Simulations. - IEEE Latin America Transactions, Vol. 12, May 2014, No 3SI, pp. 499-507.

5. En g o u 1 o u, R. G., M. B e 11 a i c h e, S. P i e r re, A. Qu in t e ro. VANET Security Surveys. Comput Commun, Vol. 44, May 2014, pp. 1-13.

6. Reyes, A., C. Barrado, M. Lopez, C. Excelente. Vehicle Density in VANET Applications. 2014, pp. 469-481.

7. Gras si, G., D. P e s ave nto, G. Pau, R. Vuy y uru, R. Wakikawa, L. Z hang. VANET via Named Data Networking. - In: 2014 IEEE Conference on Computer Communications Workshops (INFOCOM WKSHPS), 2014, pp. 410-415.

8. Zhang, H., J. Li, Y. J. L v. Structure Performance Analysis of Vehicular Ad Hoc Networks Based on Complex Network Theory. - Computer Modelling \& New Technologies, Vol. 18, April 2014, No 2, pp. 46-51.

9. M a the ew, N. M., P. C. N e e la k a n t a n. Analyzing the Network Connectivity Probability of a Linear VANET in Nakagami Fading Channels. - In: Lecture Notes in Computer Science. M. Chatterjee, K. Kothapalli, J. N. Cao and S. Rajsbaum, Eds. 2014, pp. 505-511.

10. Z h a n g, H., J. L i. Topology Analysis of VANET Based on Complex Network. - In: Proc. of 2014 International Conference on Logistics, Informatics and Service Science. Z. Zhang, Z. M. Shen, J. Zhang and R. Zhang, Eds. Berkeley, California, USA, Springer, 2014, pp. $970-974$.

11. New ma n, M. E. J. The Structure and Function of Networks. - Comput. Phys. Commun., Vol. 147, August 2002, No 1-2, pp. 40-45.

12. Z hang, H., J. Li. Dynamical Topology Analysis of VANET Based on Complex Networks Theory. - Cybernetics and Information Technologies, Vol. 14, November 2014, No 3, pp.172-186.

13. Cu o m o, F., I. R u b i n, A. B a i o c c hi, P. S a lvo. Enhanced VANET Broadcast Throughput Capacity via a Dynamic Backbone Architecture. - AD HOC Networks, Vol. 21, October 2014, pp. 42-59.

14. Z ho u, S., R. J. M o n d r a g o n. Accurately Modeling the Internet Topology. - In: Phys. Rev. E, Vol. 70, December 2004, No 06610862.

15. N e w m a n, M. E. J. Assortative Mixing in Networks. - Phys. Rev. Lett., Vol. 89, November 2002, No 20870120.

16. N e w m a n, M. E. J. A Measure of Betweenness Centrality Based on Random Walks. - Social Networks, Vol. 27, January 2005, No 1, pp. 39-54.

17. Y a n, T., W. Z h a n g, G. W a n g. DOVE: Data Dissemination to a Desired Number of Receivers in VANET. - IEEE T Veh. Technol., Vol. 63, May 2014, No 4, pp.1903-1916.

18. Doyle, J. C., D. L. Alderson, L. Li, S. Low, M. Roughan, S. Shalunov, R. T a n a ka, W. W ill ing e r. The "Robust Yet Fragile" Nature of the Internet. - P. Natl. Acad. Sci., USA, Vol. 102, October 2005, No 41, pp.14497-14502.

19. Z h u, X., D. H u, Z. H o u, L. Din g. A Location Privacy Preserving Solution to Resist Passive and Active Attacks in VANET. - China Communications, Vol. 11, September 2014, No 9, pp. 67-74. 